\title{
Inelastic silicon-hydrogen collision data for non-LTE applications in stellar atmospheres ${ }^{\star}$
}

\author{
Andrey K. Belyaev ${ }^{1,2}$, Svetlana A. Yakovleva ${ }^{2}$, and Paul S. Barklem ${ }^{1}$ \\ 1 Theoretical Astrophysics, Department of Physics and Astronomy, Uppsala University, Box 516, 75120 Uppsala, Sweden \\ e-mail: andrey.k. belyaev@gmail.com \\ 2 Department of Theoretical Physics and Astronomy, Herzen University, 191186 St. Petersburg, Russia
}

Received 30 July 2014 / Accepted 17 September 2014

\begin{abstract}
Aims. Inelastic processes in low-energy $\mathrm{Si}+\mathrm{H}$ and $\mathrm{Si}^{+}+\mathrm{H}^{-}$collisions are treated for the states from the ground state up to the ionic state, in order to provide rate coefficients needed for non-LTE modeling of Si in cool stellar atmospheres.

Methods. Electronic molecular structure is determined using a recently proposed model approach based on an asymptotic method in combination with available ab initio potentials. Nonadiabatic nuclear dynamics are treated by means of a combination of multichannel formulas and the branching-probability-current method, based on the Landau-Zener model for nonadiabatic transition probabilities. Results. Cross sections and rate coefficients for inelastic processes in $\mathrm{Si}+\mathrm{H}$ and $\mathrm{Si}^{+}+\mathrm{H}^{-}$collisions for all transitions between 26 low-lying states plus the ionic state are calculated. It is shown that the highest rate coefficient values correspond to the excitation, deexcitation, ion-pair formation, and mutual neutralization processes involving the $\mathrm{Si}\left(3 \mathrm{p} 4 \mathrm{p}{ }^{3} \mathrm{D}\right), \mathrm{Si}\left(3 \mathrm{p} 3 \mathrm{~d}{ }^{3} \mathrm{~F}\right), \operatorname{Si}\left(3 \mathrm{p} 4 \mathrm{p}{ }^{1} \mathrm{D}\right), \operatorname{Si}\left(3 \mathrm{p} 3 \mathrm{~d}{ }^{3} \mathrm{P}\right)$, $\mathrm{Si}\left(3 \mathrm{p} 4 \mathrm{p}{ }^{1} \mathrm{~S}\right)$, and the ionic $\mathrm{Si}^{+}+\mathrm{H}^{-}$states. These processes are likely to be important in non-LTE modeling.
\end{abstract}

Key words. atomic data - line: formation - stars: abundances

\section{Introduction}

Non-local thermodynamic equilibrium (non-LTE) modeling of stellar spectra is important for many fundamental problems in modern astrophysics. A non-LTE study requires detailed and complete information about the radiative and inelastic collision processes that affect the statistical equilibrium for a given atomic species. In the atmospheres of stars around solar temperature, FGK stars, the most important collisions are those with electrons and with hydrogen, where hydrogen is becoming increasingly important with decreasing metallicity. Atomic collisions are a significant source of uncertainty for non-LTE studies (see, e.g., Barklem 2012, for a recent review).

Considerable progress has recently been made in detailed quantum treatments of inelastic processes in collisions of hydrogen atoms and negative ions with atoms and positive ions of different chemical elements. Accurate quantum cross sections were calculated for transitions between many low-lying atomic and ionic states for $\mathrm{Na}, \mathrm{Li}, \mathrm{Mg}+\mathrm{H}$, as well as $\mathrm{Na}^{+}, \mathrm{Li}^{+}, \mathrm{Mg}^{+}$ $+\mathrm{H}^{-}$collisions (Belyaev et al. 1999, 2010, 2012; Croft et al. 1999a; Belyaev \& Barklem 2003; Guitou et al. 2011; Rodionov et al. 2014) based on accurate ab initio or pseudopotential electronic molecular structure calculations to provide the required quantum-chemical data (potentials and couplings). The excitation, de-excitation, ion-pair formation, and mutual neutralization processes have been studied in detail. The resulting quantum cross sections were used for computing the inelastic rate coefficients (Croft et al. 1999b; Barklem et al. 2003, 2010, 2012) and finally for non-LTE astrophysical applications (Barklem et al. 2003; Lind et al. 2009, 2011; Mashonkina 2013). It has been

\footnotetext{
* Tables 2-11 are only available at the CDS via anonymous ftp to cdsarc.u-strasbg. fr $(130.79 .128 .5)$ or via http: //cdsarc.u-strasbg.fr/viz-bin/qcat?]/A+A/572/A103
}

shown that inelastic atomic collisions of the studied elements with hydrogen are important for non-LTE modeling, leading to differences in interpreted abundances as large as 0.2 dex (see above references, or again Barklem 2012, for a review).

Silicon abundances in cool stars are of considerable astrophysical interest. Silicon is an $\alpha$-element and thus an important test case for supernova and chemical evolution models, in turn providing constraints on the initial mass function and star formation rates. It is an important opacity source and electron donor in cool stars and is also used as a reference element for comparison of various types of cosmic matter with the Sun (see, e.g., Shi et al. 2009, 2012, and references therein). Silicon abundances in very metal-poor stars are useful for comparison with supernova models (e.g., Cohen et al. 2007) and studying the role of silicate dust in low-mass star formation at early times (e.g., Ji et al. 2014).

For silicon, however, as well as for many atoms of interest, quantum cross sections for inelastic collisions with hydrogen atoms are still not available. For this reason, the socalled Drawin formula (Steenbock \& Holweger 1984; Lambert 1993), which is an extension (Drawin 1968, 1969; Drawin \& Emard 1973) of the classical Thomson model for ionization, is still widely employed for estimates of inelastic collision rate coefficients, in particular, for silicon (Shi et al. 2009, 2012; Bergemann et al. 2013, see also references therein). It is now known (Barklem et al. 2011) that in case of low-energy atomic collisions, the Drawin formula is not reliable: (i) it does not have a correct physical background; (ii) it typically overestimates inelastic rates by up to several orders of magnitude for optically allowed atomic transitions and underestimates rates for optically forbidden transitions; and (iii) it provides incorrect relative efficiencies for different transitions. Scaling factors do not improve rates obtained by means of the Drawin formula, since even if a 
scaling factor gives the correct value for one transition, it does not provide reliable rates for other transitions. Obviously, using the Drawin formula is not physical. Even more, the Drawin formula is not applicable to charge transfer processes (ion-pair formation and mutual neutralization processes), which have been found to be the most important processes in the cases studied so far (e.g. Barklem et al. 2003) ${ }^{1}$.

For these reasons, Barklem et al. (2011) emphasized the importance of deriving an approximate approach to inelastic atomic collisions with hydrogen atoms, an approach that would be physically reliable, but not as laborious or computationally expensive as a complete quantum study. Such a model approach has recently been proposed by Belyaev (2013a) based on a semiempirical asymptotic method for determination of molecular potential energies and on the branching-probability-current method for the nonadiabatic nuclear dynamics. The approach has been applied to $\mathrm{Al}+\mathrm{H}$ collisions (Belyaev 2013b). The main goal of the approach is to calculate inelastic rate coefficients with large values and estimate rate coefficients with moderate values, that is, rates of probable astrophysical interest.

In the present paper, the model approach in combination with the multichannel formulas (Belyaev \& Tserkovnyi 1987; Belyaev 1993; Belyaev \& Barklem 2003) is applied to lowenergy inelastic $\mathrm{Si}+\mathrm{H}$ and $\mathrm{Si}^{+}+\mathrm{H}^{-}$collisions of interest for stellar spectrum modelling.

\section{Model approach}

\subsection{Model description}

Inelastic processes in $\mathrm{Si}+\mathrm{H}$ and $\mathrm{Si}^{+}+\mathrm{H}^{-}$collisions are treated within the Born-Oppenheimer formalism, which is the most widely used and reliable approach for theoretical studies of lowenergy heavy-particle collisions. $\mathrm{SiH}$ ab initio potential energies have been calculated for only a few of the lowest lying states in different molecular symmetries (Kalemos et al. 2002). Nonadiabatic coupling matrix elements have not been calculated, and this prevents performing a detailed, full quantum study of these collisions. On the other hand, known adiabatic potentials allow one to estimate inelastic cross sections and rate coefficients by means of the model approach (Belyaev 2013a) based on the branching-probability-current method for the nonadiabatic nuclear dynamics. Since the treated inelastic processes are determined by nonadiabatic transitions, which are quantum by nature, the model approach is essentially quantum as well.

The quantum branching-probability-current method assumes that a nonadiabatic transition in each nonadiabatic region can be treated within the Landau-Zener (LZ) model (Landau 1932a,b; Zener 1932). Within this model, a nonadiabatic transition probability $p_{j k}$ after a single traverse of a nonadiabatic region (created by molecular states $j$ and $k$ ) can be evaluated by means of the LZ formula

$p_{j k}^{L Z}=\exp \left(-\xi_{j k} / v\right)$,

where $\xi_{j k}$ is the LZ parameter in the treated region, and $v$ the radial velocity of colliding atoms. All values are evaluated at the center of the corresponding nonadiabatic region. Positions

\footnotetext{
1 The ion-pair formation (charge transfer) process should not be confused with the ionization process. Since the physical mechanisms of these processes are different (transitions between stationary electronic states for charge transfer versus transitions between stationary and quasi-stationary states for ionization), rates for ionization processes cannot be used as estimates for charge transfer rates.
}

of nonadiabatic regions $R_{\mathrm{c}}$ are determined by local minima of adiabatic splittings $Z_{j k}(R)=\left|U_{j}-U_{k}\right|$ between adjacent adiabatic states $(k=j \pm 1)$, where $U_{j, k}(R)$ are adiabatic potentials. Several nonadiabatic regions between low-lying $\mathrm{SiH}$ molecular states at relatively short internuclear distances are found in this way based on the ab initio adiabatic potentials. In each nonadiabatic region, the $\mathrm{LZ}$ parameters can be calculated by the socalled adiabatic-potential-based formula (Belyaev \& Lebedev 2011; Belyaev 2013a):

$$
\xi_{j k}=\left.\frac{\pi}{2 \hbar} \sqrt{\frac{Z_{j k}^{3}}{Z_{j k}^{\prime \prime}}}\right|_{R=R_{\mathrm{c}}}
$$

Primed quantities refer to derivatives with respect to the internuclear distance $R$. Equation (2) expresses the LZ parameter in terms of only the adiabatic splitting $Z_{j k}$ and its second distance derivative at $R_{\mathrm{c}}$. The branching-probability-current method (Belyaev 2013a) branches each probability current after a single traverse of each nonadiabatic region according to a nonadiabatic transition probability (1) and finally allows one to calculate the inelastic transition probability $P_{i f}$ for a transition $i \rightarrow f$ taking into account many nonadiabatic transitions $j \rightarrow k$ existing between considered molecular states in any order that the transitions appear during a collision.

Previous studies of inelastic heavy particle - hydrogen collisions (Belyaev et al. 1999, 2010, 2012; Croft et al. 1999a; Belyaev \& Barklem 2003; Belyaev 2013a; Rodionov et al. 2014) show that the most important processes are ion-pair production and excitation processes due to long-range ionic-covalent interactions, and they involve the ionic and excited covalent states. In these cases, the few lowest adiabatic potential energies are not sufficient, and higher-lying potentials must be included. Longrange adiabatic potential energies can be estimated by means of the semi-empirical asymptotic method (Olson et al. 1971) within the model approach proposed in Belyaev (2013a). Since the ionic $\mathrm{Si}^{+}+\mathrm{H}^{-}$state $\left(\mathrm{Si}^{+}\right.$in the ground state) exists in the ${ }^{2} \Sigma^{+}$and ${ }^{2} \Pi$ molecular symmetries and spin-orbit couplings can be neglected (see Kalemos et al. 2002, and references therein), only the ${ }^{2} \Sigma^{+}$and ${ }^{2} \Pi$ molecular states are included in the present consideration, and nonadiabatic transitions within each of these symmetries are treated separately.

The model approach allows one to take long-range nonadiabatic regions due to ionic-covalent interactions into account. In this case, nonadiabatic regions are passed by the system in a particular order, and although the branching-probability-current method also covers this situation, one can use the multichannel model (Belyaev \& Tserkovnyi 1987; Belyaev 1993; Belyaev \& Barklem 2003) to treat the nonadiabatic nuclear dynamics. This model, if applicable, is advantageous because it is analytical in contrast to the branching-probability-current method, which is numerical.

Within the multichannel model, the probability $P_{\text {if }}$ for a transition from a given initial state $i$ to a given final state $f$ is determined by the following multichannel formulas. For mutual neutralization, the formula reads as (Belyaev \& Tserkovnyi 1987; Belyaev 1993)

$P_{i f}=2 p_{f}\left(1-p_{f}\right)\left\{\prod_{l=f+1}^{F} p_{l}\right\}\left\{1+\sum_{m=1}^{2(f-1)} \prod_{k=1}^{m}\left(-p_{f-[(k+1) / 2]}\right)\right\}$, 
and for de-excitation the formula is (Belyaev \& Barklem 2003)

$P_{i f}=2 p_{f}\left(1-p_{f}\right)\left(1-p_{i}\right)\left\{\prod_{l=f+1}^{i-1} p_{l}\right\}\left\{1+\sum_{m=1}^{2(f-1)} \prod_{k=1}^{m}\left(-p_{f-[(k+1) / 2]}\right)\right\}$,

where $F$ is the total number of nonadiabatic regions created by one ionic and $F$ covalent states, with square brackets [...] in the subscript denoting the highest integer value. Labeling of adiabatic molecular states and, hence, nonadiabatic regions and nonadiabatic transition probabilities in Eqs. (1) and (2) is done separately in each molecular symmetry since only nonadiabatic transitions between adjacent adiabatic states of the same symmetry are taken into account. For this reason, labelings differ in the ${ }^{2} \Sigma^{+}$and ${ }^{2} \Pi$ symmetries. At present, the adiabatic molecular states in each symmetry are labeled from the bottom to the top (see below) in contrast to (Belyaev \& Tserkovnyi 1987; Belyaev 1993), where they are labeled from the top to the bottom. The nonadiabatic transition probability $p_{j}$ at a single nonadiabatic region is determined by means of the $\mathrm{LZ}$ model

$p_{j} \equiv p_{j j+1}=p_{j j+1}^{L Z}$,

see Eqs. (1) and (2). Again, Eqs. (1) and (2) allow one to calculate nonadiabatic transition probabilities in each nonadiabatic region based on information about adiabatic potentials alone, while Eqs. (3) and (4) give inelastic transition probabilities for exothermal processes.

When the collisional energy is below the energy thresholds for channels lying higher, it is necessary to take into account that every nonadiabatic region is passed several times owing to oscillations in an ionic-diabatic potential. In this case the nonadiabatic transition probability can be expressed as a sum of an infinite geometric progression as described by Belyaev (1985). This gives some corrections to Eq. (4), which are also considered at present.

Inelastic cross sections and rate constants are determined as usual

$\sigma_{i f}(E)=\frac{\pi \hbar^{2} p_{i}^{\text {stat }}}{2 \mu E} \sum_{J=0}^{J_{\max }} P_{i f}(J, E)(2 J+1)$,

$K_{i f}(T)=\sqrt{\frac{8}{\pi \mu\left(k_{\mathrm{B}} T\right)^{3}}} \int_{0}^{\infty} \sigma_{i f}(E) E \exp \left(-\frac{E}{k_{\mathrm{B}} T}\right) \mathrm{d} E$

where $p_{i}^{\text {stat }}$ is the statistical probability for the population of the initial channel $i$. Other notations are as usual. For the inverse (endothermic) processes, cross sections and rate coefficients are calculated via the detailed balance relations:

$\sigma_{f i}(E)=\sigma_{i f}\left(E+\Delta E_{f i}\right) \frac{p_{f}^{\text {stat }}}{p_{i}^{\text {stat }}} \frac{E+\Delta E_{f i}}{E}$

$K_{f i}(T)=K_{i f}(T) \frac{p_{f}^{\text {stat }}}{p_{i}^{\text {stat }}} \exp \left(\frac{\Delta E_{f i}}{k_{\mathrm{B}} T}\right)$

$\Delta E_{f i}=E_{f}-E_{i}$ being the energy defect of the process ${ }^{2}$.

\footnotetext{
2 Note that $\Delta E_{f i}$ is negative for an endothermic process $f \rightarrow i$.
}

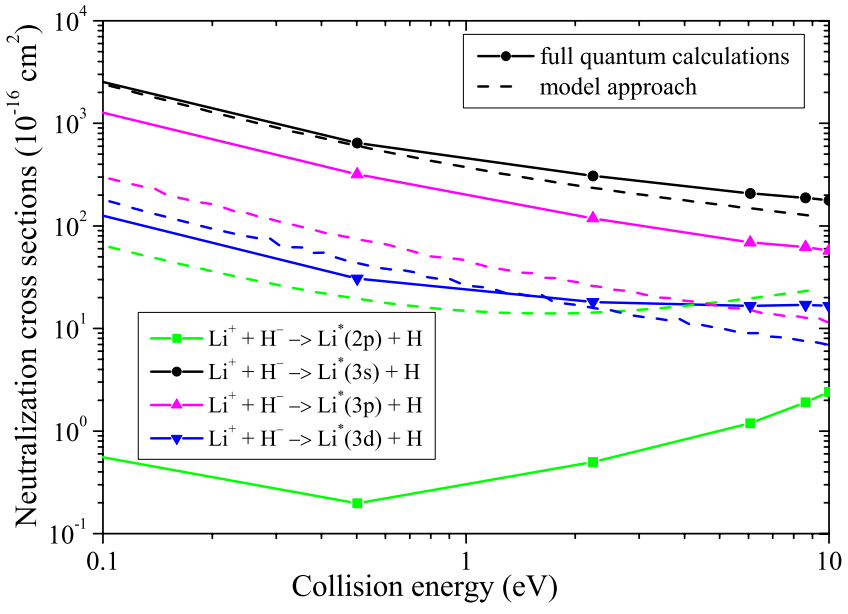

Fig. 1. Comparison of $\mathrm{Li}^{+}+\mathrm{H}^{-}$mutual neutralization cross sections. Solid lines represent the full quantum calculations (Croft et al. 1999a); dashed ones the present model approach.

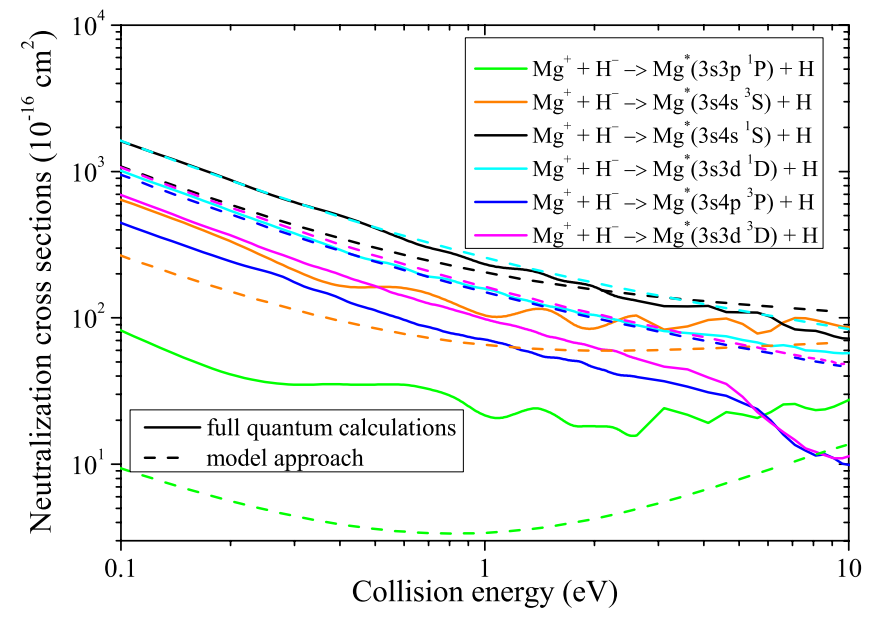

Fig. 2. Comparison of $\mathrm{Mg}^{+}+\mathrm{H}^{-}$mutual neutralization cross sections. Solid lines represent the full quantum calculations (Belyaev et al. 2012; Rodionov et al. 2014); dashed ones the present model approach.

\subsection{Model tests}

Before applying the model approach described above to $\mathrm{Si}+\mathrm{H}$ and $\mathrm{Si}^{+}+\mathrm{H}^{-}$collisions, the approach was tested for the mutual neutralization processes. Cross sections were calculated for $\mathrm{Li}^{+}+\mathrm{H}^{-}$and $\mathrm{Mg}^{+}+\mathrm{H}^{-}$collisions using asymptotic potentials and the multichannel formula in order to compare them with the cross sections obtained by full quantum calculations (Croft et al. 1999a; Belyaev et al. 2012; Rodionov et al. 2014). It is known that mutual neutralization processes are important in astrophysical applications.

Figures 1 and 2 compare the model and the full quantum mutual neutralization cross sections in $\mathrm{Li}^{+}+\mathrm{H}^{-}$and $\mathrm{Mg}^{+}+$ $\mathrm{H}^{-}$collisions, respectively. It is seen that the cross sections with the highest values (corresponding to the final states $\mathrm{Li}(3 \mathrm{~s})$ and $\operatorname{Mg}\left(3 \mathrm{~s} 4 \mathrm{~s}{ }^{1} \mathrm{~S}\right)$, respectively) are in good agreement, approximately within a factor of 2 . The cross sections with moderate values agree within a factor of 2 to 10 . These two groups of processes (with high and moderate values of cross sections) are those of likely importance in astrophysical applications. Cross sections with relatively low values (in particular, involving the ground states) differ noticeably from the full quantum results 
owing to large potential energy splittings in short-range nonadiabatic regions, which are poorly described by the asymptotic method. This group of processes is expected to be unimportant in applications. Using accurate adiabatic potentials instead of model estimates in combination with the branching-probabilitycurrent method instead of the multichannel formulas improves agreement with the full quantum cross sections. These findings agree with the conclusions described by Belyaev (2013a).

It is worth pointing out that we consider that the comparison presented in Figs. 1 and 2 shows good agreement between the quantum model and the full quantum approaches. First, the model approach correctly identifies the dominant partial pro$\operatorname{cesses}^{3}$. Second, it provides correct values for the corresponding cross sections (those with the highest values). In addition, the model approach estimates the correct order of magnitude for processes with moderate cross sections. And finally, both the model and the full quantum approaches yield low (negligible) cross sections for other processes. The electronic structure of $\mathrm{Si}$ (studied in the present paper) is not more complicated than for $\mathrm{Mg}$ : Both elements have two valence electrons, and the appearance of more states in $\mathrm{Si}$ is due to a $3 \mathrm{p}$ valence electron instead of a $3 \mathrm{~s}$ valence electron in $\mathrm{Mg}$. Moreover, the processes treated in the present paper are mainly based on one-electron transitions, so the findings obtained for $\mathrm{Li}^{+}, \mathrm{Mg}^{+}+\mathrm{H}^{-}$collisions are expected to be valid for $\mathrm{Si}^{+}+\mathrm{H}^{-}$and $\mathrm{Si}+\mathrm{H}$ collisions.

In relation to this point, we make a general comment regarding comparing quantum mechanical data with those provided by the Drawin formula. The Drawin formula yields zero cross sections for all charge-transfer processes; i.e. the processes compared in this section, the processes for which the accurate full quantum approach gives the largest cross sections, and the processes that have been found to be the most important in astrophysical applications so far (see Barklem et al. 2011, for a detailed discussion). Thus, we see no sense in further comparisons of results with the Drawin formula, given that it does not model the largest and most important processes.

\section{Results of calculations}

\subsection{Electronic molecular structure of $\mathrm{SiH}$}

Twenty-six low-lying $\mathrm{SiH}\left({ }^{2} \Sigma^{+},{ }^{2} \Pi\right)$ molecular states and the ionic state are taken into account in the present study. They are collected in Table 1. States not leading to ${ }^{2} \Sigma^{+}$and ${ }^{2} \Pi$ symmetries may have ${ }^{2} \Sigma^{-},{ }^{2} \Delta$, etc. and also quartet molecular symmetries, but these symmetries need not be considered here since the relevant ionic state, here $j_{\text {at }}=27$, does not lead to these symmetries. For this reason, the state corresponding asymptotically to $\mathrm{Si}\left(3 \mathrm{~s} 3 \mathrm{p}^{35} \mathrm{~S}\right)+\mathrm{H}\left(1 \mathrm{~s}{ }^{2} \mathrm{~S}\right)$ is not included in the present treatment; it cannot interact with the ionic state and thus has no mechanism for inelastic processes in the present description ${ }^{4}$. Processes involving this state must therefore occur by some other mechanism, probably at short range, and cross sections can be expected to be small. There are more states asymptotically lying between the covalent $\mathrm{Si}\left(3 \mathrm{p} 4 \mathrm{~d}^{3} \mathrm{~F}\right)+\mathrm{H}\left(1 \mathrm{~s}{ }^{2} \mathrm{~S}\right)$ state and the ionic $\mathrm{Si}^{+}\left(3 \mathrm{p}^{2} \mathrm{P}\right)+\mathrm{H}^{-}\left(1 \mathrm{~s}^{2}{ }^{1} \mathrm{~S}\right)$ state, but nonadiabatic regions for them are located at internuclear distances greater than $100 \mathrm{au}$, so adiabatic splittings in these regions are too small to

\footnotetext{
3 Partial processes are those from one atomic channel to a particular final atomic channel, as distinct from total processes from one atomic channel to all possible final atomic channels.

4 We note that this state would interact with the first excited ionic state $\mathrm{Si}^{+}\left(3 \mathrm{~s} 3 \mathrm{p}^{2}{ }^{2} \mathrm{P}\right)+\mathrm{H}^{-}\left(1 \mathrm{~s}^{2}{ }^{1} \mathrm{~S}\right)$ in the ${ }^{4} \Sigma^{-}$symmetry, but this interaction takes place at short range $(<5 \mathrm{au})$.
}

Table 1. SiH molecular channels, the corresponding asymptotic atomic states, and asymptotic energies with respect to the ground state.

\begin{tabular}{|c|c|c|c|c|}
\hline$\underline{j_{\text {at }}}$ & $\begin{array}{c}\text { Asymptotic } \\
\text { atomic states }\end{array}$ & $\begin{array}{r}\text { Mol } \\
\text { sym } \\
\end{array}$ & $\begin{array}{l}\text { cular } \\
\text { ietries }\end{array}$ & $\begin{array}{l}\text { Asymptotic } \\
\text { energies }(\mathrm{eV})\end{array}$ \\
\hline 1 & $\mathrm{Si}\left(3 \mathrm{p}^{2}{ }^{3} \mathrm{P}\right)+\mathrm{H}\left(1 \mathrm{~s}^{2} \mathrm{~S}\right)$ & & ${ }^{2} \Pi$ & 0.0 \\
\hline 2 & $\mathrm{Si}\left(3 \mathrm{p}^{2}{ }^{1} \mathrm{D}\right)+\mathrm{H}\left(1 \mathrm{~s}^{2} \mathrm{~S}\right)$ & ${ }^{2} \Sigma^{+}$ & ${ }^{2} \Pi$ & 0.78096 \\
\hline 3 & $\mathrm{Si}\left(3 \mathrm{p}^{2}{ }^{1} \mathrm{~S}\right)+\mathrm{H}\left(1 \mathrm{~s}^{2} \mathrm{~S}\right)$ & ${ }^{2} \Sigma^{+}$ & & 1.90866 \\
\hline 4 & $\mathrm{Si}\left(3 \mathrm{p} 4 \mathrm{~s}^{3} \mathrm{P}\right)+\mathrm{H}\left(1 \mathrm{~s}^{2} \mathrm{~S}\right)$ & ${ }^{2} \Sigma^{+}$ & ${ }^{2} \Pi$ & 4.94199 \\
\hline 5 & $\mathrm{Si}\left(3 \mathrm{p} 4 \mathrm{~s}{ }^{1} \mathrm{P}\right)+\mathrm{H}\left(1 \mathrm{~s}^{2} \mathrm{~S}\right)$ & ${ }^{2} \Sigma^{+}$ & ${ }^{2} \Pi$ & 5.08235 \\
\hline 6 & $\mathrm{Si}\left(3 \mathrm{~s} 3 \mathrm{p}^{3}{ }^{3} \mathrm{D}\right)+\mathrm{H}\left(1 \mathrm{~s}^{2} \mathrm{~S}\right)$ & ${ }^{2} \Sigma^{+}$ & ${ }^{2} \Pi$ & 5.61689 \\
\hline 7 & $\mathrm{Si}\left(3 p 4 p{ }^{1} \mathrm{P}\right)+\mathrm{H}\left(1 \mathrm{~s}^{2} \mathrm{~S}\right)$ & & ${ }^{2} \Pi$ & 5.86248 \\
\hline 8 & $\mathrm{Si}\left(3 \mathrm{p} 3 \mathrm{~d}^{1} \mathrm{D}\right)+\mathrm{H}\left(1 \mathrm{~s}^{2} \mathrm{~S}\right)$ & & ${ }^{2} \Pi$ & 5.87084 \\
\hline 9 & $\mathrm{Si}\left(3 \mathrm{p} 4 \mathrm{p}^{3} \mathrm{D}\right)+\mathrm{H}\left(1 \mathrm{~s}^{2} \mathrm{~S}\right)$ & ${ }^{2} \Sigma^{+}$ & ${ }^{2} \Pi$ & 5.97126 \\
\hline 10 & $\mathrm{Si}\left(3 \mathrm{p} 4 \mathrm{p}{ }^{3} \mathrm{P}\right)+\mathrm{H}\left(1 \mathrm{~s}^{2} \mathrm{~S}\right)$ & & ${ }^{2} \Pi$ & 6.09111 \\
\hline 11 & $\mathrm{Si}\left(3 \mathrm{p} 4 \mathrm{p}^{3} \mathrm{~S}\right)+\mathrm{H}\left(1 \mathrm{~s}^{2} \mathrm{~S}\right)$ & ${ }^{2} \Sigma^{+}$ & & 6.12478 \\
\hline 12 & $\mathrm{Si}\left(3 \mathrm{p} 3 \mathrm{~d}^{3} \mathrm{~F}\right)+\mathrm{H}\left(1 \mathrm{~s}^{2} \mathrm{~S}\right)$ & ${ }^{2} \Sigma^{+}$ & ${ }^{2} \Pi$ & 6.19498 \\
\hline 13 & $\mathrm{Si}\left(3 \mathrm{p} 4 \mathrm{p}{ }^{1} \mathrm{D}\right)+\mathrm{H}\left(1 \mathrm{~s}^{2} \mathrm{~S}\right)$ & ${ }^{2} \Sigma^{+}$ & ${ }^{2} \Pi$ & 6.22269 \\
\hline 14 & $\mathrm{Si}\left(3 \mathrm{p} 3 \mathrm{~d}^{3} \mathrm{P}\right)+\mathrm{H}\left(1 \mathrm{~s}^{2} \mathrm{~S}\right)$ & ${ }^{2} \Sigma^{+}$ & ${ }^{2} \Pi$ & 6.26535 \\
\hline 15 & $\mathrm{Si}\left(3 \mathrm{p} 4 \mathrm{p}{ }^{1} \mathrm{~S}\right)+\mathrm{H}\left(1 \mathrm{~s}^{2} \mathrm{~S}\right)$ & ${ }^{2} \Sigma^{+}$ & & 6.39907 \\
\hline 16 & $\mathrm{Si}\left(3 \mathrm{p} 3 \mathrm{~d}^{1} \mathrm{~F}\right)+\mathrm{H}\left(1 \mathrm{~s}^{2} \mathrm{~S}\right)$ & ${ }^{2} \Sigma^{+}$ & ${ }^{2} \Pi$ & 6.61607 \\
\hline 17 & $\mathrm{Si}\left(3 \mathrm{p} 3 \mathrm{~d}^{1} \mathrm{P}\right)+\mathrm{H}\left(1 \mathrm{~s}^{2} \mathrm{~S}\right)$ & ${ }^{2} \Sigma^{+}$ & ${ }^{2} \Pi$ & 6.61919 \\
\hline 18 & $\mathrm{Si}\left(3 \mathrm{p} 3 \mathrm{~d}^{3} \mathrm{D}\right)+\mathrm{H}\left(1 \mathrm{~s}^{2} \mathrm{~S}\right)$ & & ${ }^{2} \Pi$ & 6.72312 \\
\hline 19 & $\mathrm{Si}\left(3 \mathrm{p} 5 \mathrm{~s}^{3} \mathrm{P}\right)+\mathrm{H}\left(1 \mathrm{~s}^{2} \mathrm{~S}\right)$ & ${ }^{2} \Sigma^{+}$ & ${ }^{2} \Pi$ & 6.74788 \\
\hline 20 & $\mathrm{Si}\left(3 \mathrm{p} 5 \mathrm{~s}{ }^{1} \mathrm{P}\right)+\mathrm{H}\left(1 \mathrm{~s}^{2} \mathrm{~S}\right)$ & ${ }^{2} \Sigma^{+}$ & ${ }^{2} \Pi$ & 6.80314 \\
\hline 21 & $\mathrm{Si}\left(3 \mathrm{p} 4 \mathrm{~d}^{1} \mathrm{D}\right)+\mathrm{H}\left(1 \mathrm{~s}^{2} \mathrm{~S}\right)$ & & ${ }^{2} \Pi$ & 7.00552 \\
\hline 22 & $\mathrm{Si}\left(3 \mathrm{p} 4 \mathrm{~d}^{3} \mathrm{P}\right)+\mathrm{H}\left(1 \mathrm{~s}{ }^{2} \mathrm{~S}\right)$ & ${ }^{2} \Sigma^{+}$ & ${ }^{2} \Pi$ & 7.02975 \\
\hline 23 & $\mathrm{Si}\left(3 p 5 p{ }^{1} \mathrm{P}\right)+\mathrm{H}\left(1 \mathrm{~s}^{2} \mathrm{~S}\right)$ & & ${ }^{2} \Pi$ & 7.03988 \\
\hline 24 & $\mathrm{Si}\left(3 \mathrm{p} 5 \mathrm{p}^{3} \mathrm{D}\right)+\mathrm{H}\left(1 \mathrm{~s}^{2} \mathrm{~S}\right)$ & ${ }^{2} \Sigma^{+}$ & ${ }^{2} \Pi$ & 7.07874 \\
\hline 25 & $\mathrm{Si}\left(3 \mathrm{p} 5 \mathrm{p}{ }^{3} \mathrm{P}\right)+\mathrm{H}\left(1 \mathrm{~s}^{2} \mathrm{~S}\right)$ & & ${ }^{2} \Pi$ & 7.11702 \\
\hline 26 & $\mathrm{Si}\left(3 \mathrm{p} 4 \mathrm{~d}^{3} \mathrm{~F}\right)+\mathrm{H}\left(1 \mathrm{~s}^{2} \mathrm{~S}\right)$ & ${ }^{2} \Sigma^{+}$ & ${ }^{2} \Pi$ & 7.12772 \\
\hline 27 & $\mathrm{Si}^{+}\left(3 \mathrm{p}^{2} \mathrm{P}\right)+\mathrm{H}^{-}\left(1 \mathrm{~s}^{2}{ }^{1} \mathrm{~S}\right)$ & ${ }^{2} \Sigma^{+}$ & ${ }^{2} \Pi$ & 7.40168 \\
\hline
\end{tabular}

produce any significant inelastic transitions. For this reason the states above $\mathrm{Si}\left(3 \mathrm{p} 4 \mathrm{~d}{ }^{3} \mathrm{~F}\right)+\mathrm{H}\left(1 \mathrm{~s}{ }^{2} \mathrm{~S}\right)$ are not included into the present treatment.

We note that the atomic state $j_{\text {at }}=6$ corresponds to a two electron excitation of $\mathrm{Si}$, and a more rigorous treatment would account for this fact (e.g. see Belyaev 1993). However, as will be seen, processes involving this state are not expected to be large (and thus astrophysically important), and so we neglect this complication.

The index $j_{\text {at }}$ in Table 1 labels atomic channels (interactions between atomic particles at infinite internuclear distance) and does not coincide with labels of ${ }^{2} \Sigma^{+},{ }^{2} \Pi$ molecular states used in Eqs. (3) and (4) for the nonadiabatic nuclear dynamics since one atomic channel can generate (several) different molecular states.

Adiabatic potentials for ${ }^{2} \Sigma^{+}$and ${ }^{2} \Pi$ molecular symmetries are presented in Figs. 3 and 4, respectively. The ab initio adiabatic potentials for the four lowest ${ }^{2} \Sigma^{+}$states and for the three lowest ${ }^{2} \Pi$ states are calculated by Kalemos et al. (2002), while the higher-lying state potentials are obtained by means of the model approach described by Belyaev (2013a).

Many avoided crossings with local minima of the adiabatic potential splittings are clearly seen. Each avoided crossing yields a nonadiabatic region of the LZ type with LZ parameters that are evaluated by Eq. (2). The nonadiabatic regions can be divided into two groups: (i) short-range nonadiabatic regions between low-lying molecular states (sometimes with several avoided crossings between the same adjacent states); and (ii) a series of long-range avoided crossings due to the ionic-covalent interaction (only one such nonadiabatic region between adjacent states). For the latter, nonadiabatic regions are passed in a particular order and one can use the analytic multichannel model 


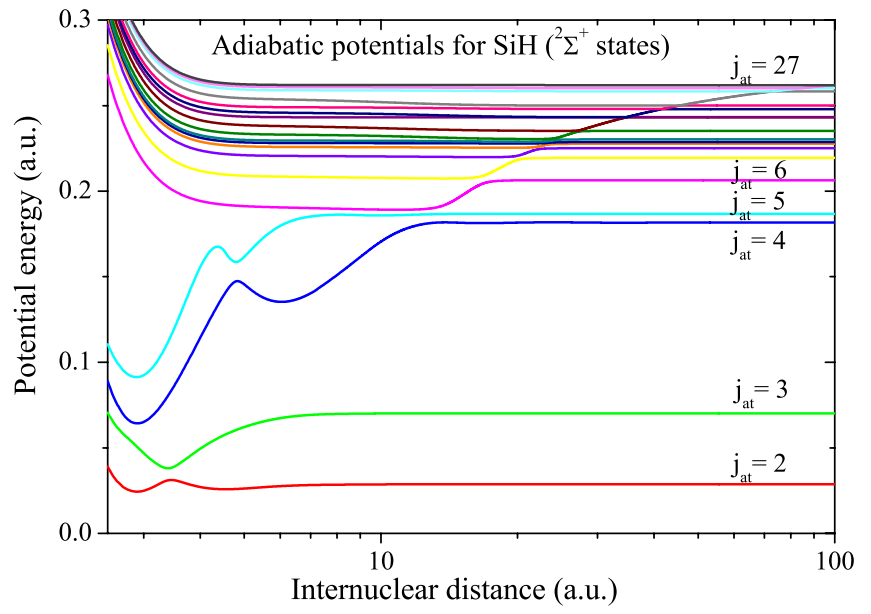

Fig. 3. Adiabatic potential energies for $\mathrm{SiH}_{(}\left({ }^{2} \Sigma^{+}\right)$states. The four loweststate potentials are the accurate ab initio data (Kalemos et al. 2002), other potentials are estimated by the asymptotic approach. The labels $j_{\text {at }}$ correspond to the atomic channels in Table 1.

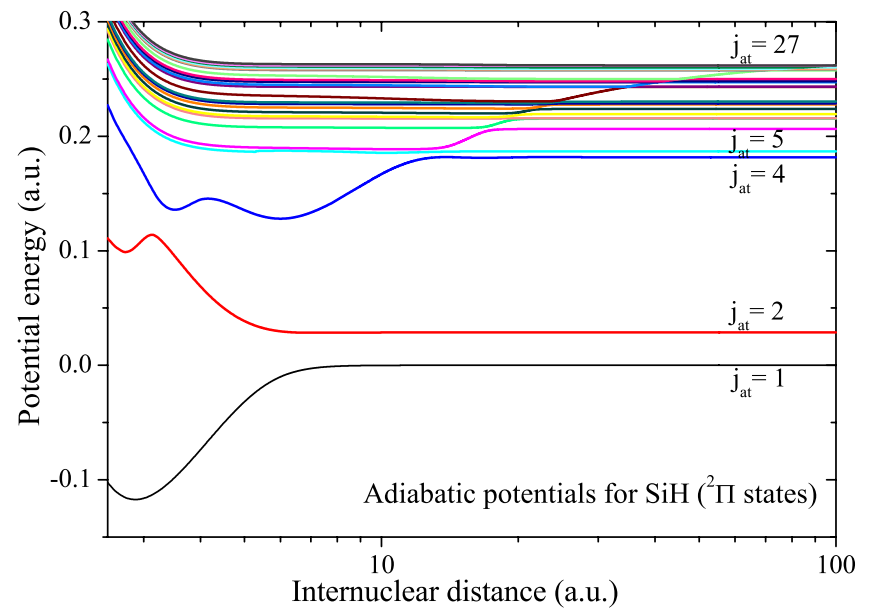

Fig. 4. Adiabatic potential energies for $\operatorname{SiH}\left({ }^{2} \Pi\right)$ states. The four loweststate potentials are the accurate ab initio data (Kalemos et al. 2002), other potentials are estimated by the asymptotic approach. The labels $j_{\text {at }}$ correspond to the atomic channels in Table 1.

to treat the nonadiabatic nuclear dynamics, while for the former there is no particular order of nonadiabatic regions passed by the system, so one has to use the branching-probability-current method or something similar. Tests show that the results of using these two methods are different only in the ${ }^{2} \Sigma^{+}$symmetry, but not in the ${ }^{2} \Pi$ symmetry. Thus, the branching-probability-current method is applied for transitions between low-lying states only in the ${ }^{2} \Sigma^{+}$symmetry.

\subsection{Cross sections and rate coefficients}

Inelastic cross sections and rate coefficients are calculated as described above for all transitions between the 27 states in Table 1, including the ionic state. The transitions are treated separately in ${ }^{2} \Sigma^{+}$and ${ }^{2} \Pi$ molecular symmetries with proper inclusion of statistical probabilities. Final cross sections and rate coefficients are obtained as a sum of partial values over possible molecular symmetries.

There are many inelastic processes with substantial values for cross sections and rate coefficients in $\mathrm{Si}+\mathrm{H}$ and $\mathrm{Si}^{+}+\mathrm{H}^{-}$ collisions. A plot showing the magnitude of all rate coefficients for inelastic $\mathrm{Si}+\mathrm{H}$ collisions at temperature $T=6000 \mathrm{~K}$ is shown in Fig. 5.

It is seen that there are a few processes with rate coefficients exceeding $10^{-8} \mathrm{~cm}^{3} / \mathrm{s}$ and relatively many processes with rate coefficients exceeding $10^{-9} \mathrm{~cm}^{3} / \mathrm{s}$. These are the processes of mutual neutralization into the $\operatorname{Si}\left(3 p 4 p{ }^{3} D\right), \operatorname{Si}\left(3 p 4 p{ }^{3} P\right)$, $\operatorname{Si}\left(3 p 3 d{ }^{3} F\right), \operatorname{Si}\left(3 p 4 p{ }^{1} D\right), \operatorname{Si}\left(3 p 3 d{ }^{3} P\right), \operatorname{Si}\left(3 p 4 p{ }^{1} S\right)+H$ final states, their inverse processes, the ion-pair formation ones from these states, as well as excitation and de-excitation processes from and to these states. It is expected that these processes are prime candidates to be important in astrophysical applications, although some inelastic processes involving other states also have significant rate coefficients and might be important in applications as well. It is also seen that for many processes, especially involving the lowest-lying states, rate coefficients have negligible values. These processes are expected to be unimportant in applications.

It is worth noting that many partial inelastic processes are based on nonadiabatic transitions within both the ${ }^{2} \Sigma^{+}$and ${ }^{2} \Pi$ symmetries; in this case cross sections and rate coefficients are determined as a sum of corresponding values over these two symmetries. Some other processes occur only in one of these symmetries; in this case, no summation is performed. And finally, for a few partial processes, the initial and final channels provide molecular states of different symmetries. An example is an excitation process from the atomic channel $j_{\text {at }}=3$, which generates only the ${ }^{2} \Sigma^{+}$molecular state, to the atomic channel $j_{\text {at }}=7$, which produces no ${ }^{2} \Sigma^{+}$molecular state, but has the ${ }^{2} \Sigma^{-}$ and ${ }^{2} \Pi$ states. For such partial processes, the present model approach gives zero cross sections and rate coefficients. Other couplings, e.g. spin-orbit ones, if taken into account, provide negligible inelastic cross sections and rate coefficients as compared to those obtained within the ${ }^{2} \Sigma^{+}$and ${ }^{2} \Pi$. This explains the zero rate coefficients seen in Fig. 5 for some partial processes.

The tabulated data for the calculated rate coefficients are available upon request and accessible online at the CDS for selected temperatures $T$, containing the following information. In each file for a given $T$, column 1 lists the initial state of $\mathrm{Si}$ in collisions with $\mathrm{H}$ or the ionic state $\mathrm{Si}^{+}+\mathrm{H}^{-}$, Columns 2-28 give the matrices of the inelastic rate coefficients $K_{i f}(T)$.

Among the exothermic processes, the highest value for the rate coefficient corresponds to the mutual neutralization process into the $\mathrm{Si}\left(3 \mathrm{p} 3 \mathrm{~d}^{3} \mathrm{P}\right)+\mathrm{H}$ final state, as well as de-excitation processes from and to this state. The energy dependence of cross sections and the temperature dependence of rate coefficients for the mutual neutralization, the ion-pair formation, the excitation, and the de-excitation processes from and to the $\mathrm{Si}\left(3 \mathrm{p} 3 \mathrm{~d}^{3} \mathrm{P}\right)+\mathrm{H}$ state are shown in Figs. 6 and 7. Such dependences are typical for these processes (see, e.g., Belyaev 2013a).

Analysis of the calculated cross sections and rate coefficients shows that the inelastic processes with high to moderate values are determined by several long-range nonadiabatic regions, where the asymptotic method provides rather accurate adiabatic potentials and the use of the LZ model is justified. In this case, the nonadiabatic transition probabilities $p_{j k}^{\mathrm{LZ}}$ are expected to be accurate and, thus, if multiple transitions (a multichannel case) are taken into account, inelastic transition probabilities $P_{\text {if }}$ are also expected to be accurate. Multiple transitions may be accounted for by means of the multichannel formulas or the branching-probability-current method, both of which account for the fact that probability currents pass several nonadiabatic regions during a collision. Finally, the calculated inelastic cross sections and rate coefficients with high and moderate 

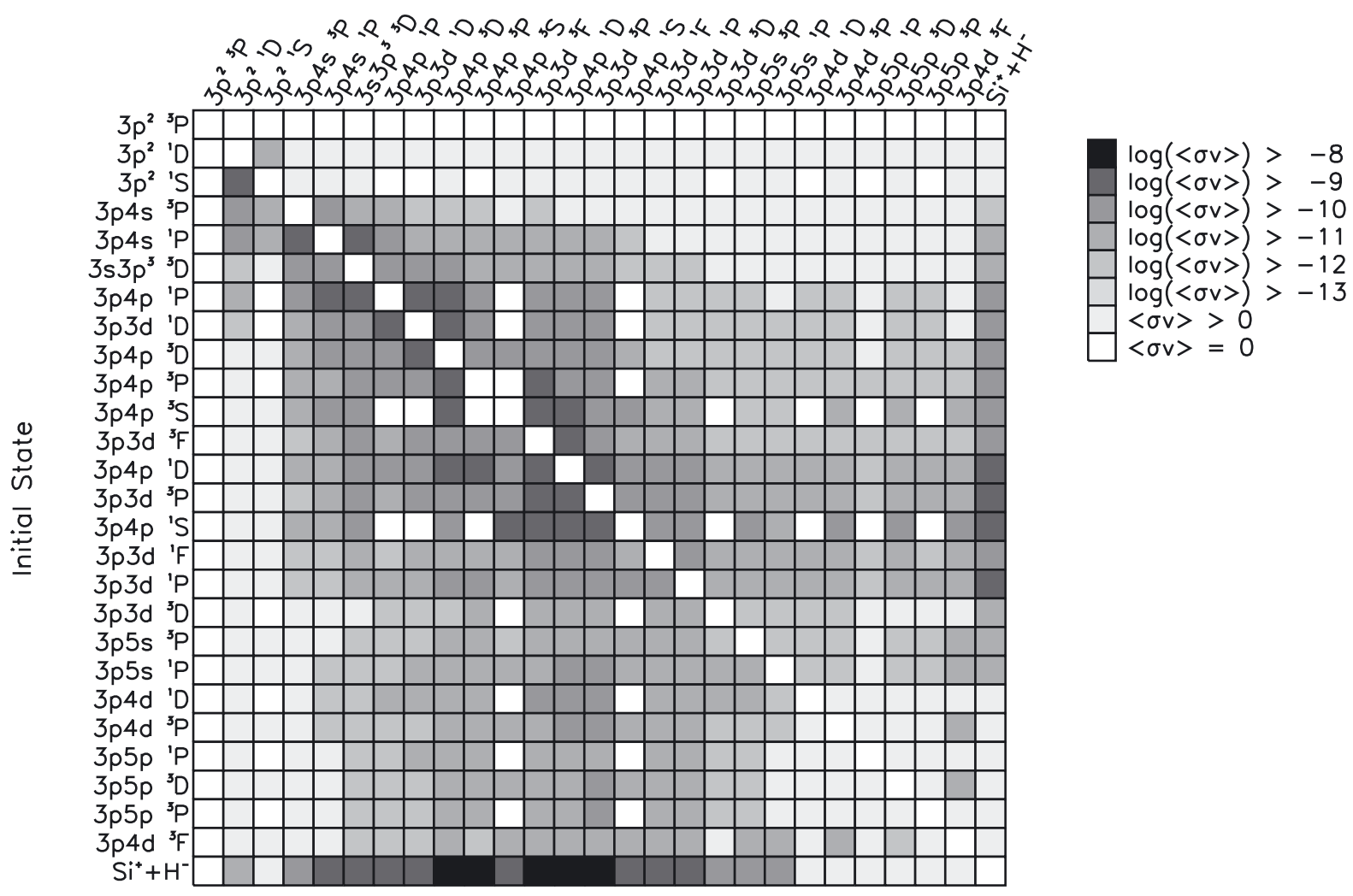

Fig. 5. Graphical representation of the rate coefficient matrix $\langle\sigma v\rangle\left(\mathrm{in} \mathrm{cm}^{3} / \mathrm{s}\right)$ for inelastic $\mathrm{Si}+\mathrm{H}$ and $\mathrm{Si}^{+}+\mathrm{H}^{-}$collisions at temperature $T=$ $6000 \mathrm{~K}$.

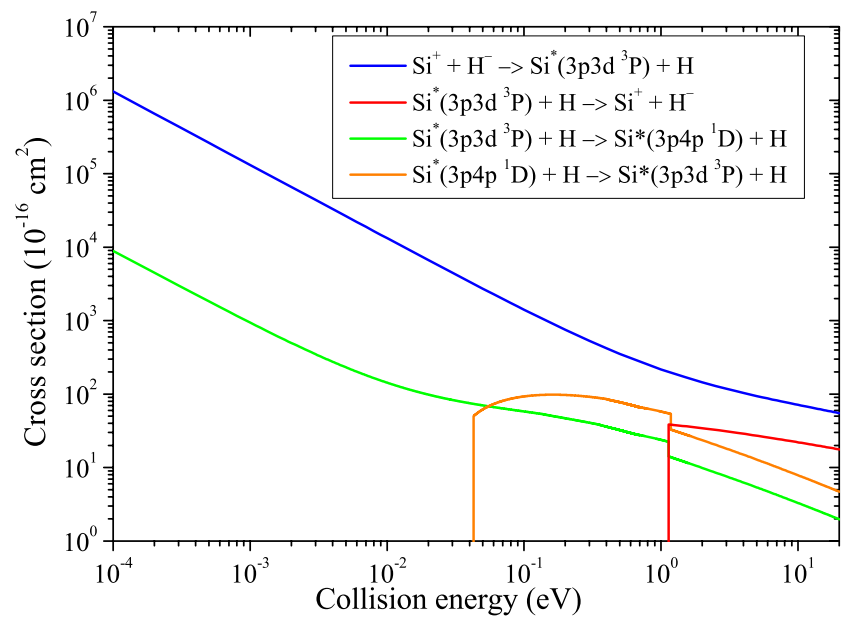

Fig. 6. Inelastic cross sections for mutual neutralization, ion-pair formation, de-excitation, and excitation to and from the $\mathrm{Si}\left(3 \mathrm{p} 3 \mathrm{~d}^{3} \mathrm{P}\right)+\mathrm{H}$ state.

values are also expected to be obtained with reasonable accuracy. (The expected accuracy is discussed further below.)

\section{Discussion and conclusion}

Cross sections and rate coefficients for inelastic processes in low-energy $\mathrm{Si}+\mathrm{H}$ and $\mathrm{Si}^{+}+\mathrm{H}^{-}$collisions have been calculated for all transitions between 26 low-lying states, as well as the ionic state, in the ${ }^{2} \Sigma^{+}$and ${ }^{2} \Pi$ molecular symmetries. The adiabatic potentials were constructed from the available

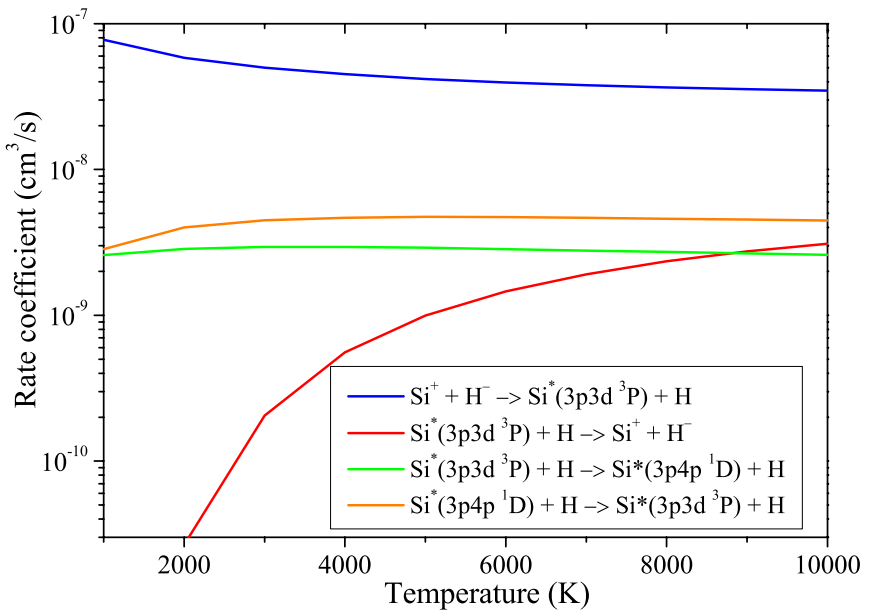

Fig. 7. Rate coefficients for the same processes as in Fig. 6.

$\mathrm{ab}$ initio data for the lowest states and from the asymptotic model estimates for higher-lying states including the ionic one. The branching-probability-current method in combination with the multichannel model is used for nonadiabatic nuclear dynamical calculations.

Analysis of the calculated data allows one to divide the treated processes into three groups. The first group consists of processes with high values of cross sections and rate coefficients. The largest cross sections and rate coefficients (up to roughly $100 \AA^{2}$ and $8 \times 10^{-9} \mathrm{~cm}^{3} / \mathrm{s}$, respectively, for endothermic processes) correspond to the excitation, de-excitation, ion-pair formation, and mutual neutralization processes involving the 
$\operatorname{Si}\left(3 p 4 p^{3} D\right), \operatorname{Si}\left(3 p 4 p^{3} P\right), \operatorname{Si}\left(3 p 3 d^{3} F\right), \operatorname{Si}\left(3 p 4 p{ }^{1} D\right), \operatorname{Si}\left(3 p 3 d^{3} P\right)$, $\mathrm{Si}\left(3 \mathrm{p} 4 \mathrm{p}{ }^{1} \mathrm{~S}\right)$, and the ionic states. These inelastic processes are based on multiple nonadiabatic transitions involving several nonadiabatic regions due to the ionic-covalent interaction at large internuclear distances. The parameters of the longrange nonadiabatic regions are well defined by the model, so the calculated estimates for the corresponding cross sections and rate coefficients are expected to be accurate within a factor of 2 . This estimate comes from comparing the quantum and the model calculations of cross sections for lithium- and magnesiumhydrogen collisions (see Sect. 2.2), as well as from previous experience of applying the asymptotic formula of Olson et al. (1971). The inelastic processes involving the states from this group are expected to be important for astrophysical application in non-LTE modeling. As has been seen in earlier work, increased coupling between the neutral and ionized species via charge transfer processes (mutual neutralization and ion-pair production) generally lessens non-LTE effects (Barklem et al. 2003; Lind et al. 2009, 2011). In the case of Li (Barklem et al. 2003 ) it was shown that a factor of 2 error in such processes led only to very small effects in abundances (of order 1\%), and thus we expect the accuracy of the data computed here to be sufficient. Increased coupling between different atomic spin systems, here singlet and triplet atomic states, via spin-changing collisions has less predictable effects on non-LTE models, but has been found to have significant effects on $\mathrm{O}$ in the case of electron collisions (Barklem 2007; Fabbian et al. 2009) and on $\mathrm{Mg}$ (Osorio et al., in prep.) in the case of both electron and hydrogen collisions.

The second group includes processes with moderate cross sections and rate coefficients, which are due to several intermediate to long range nonadiabatic regions formed by the ioniccovalent interaction. The estimates for this group are believed to be reliable within a factor of $2-10$. The processes from this group could potentially have some effects in astrophysical applications.

The third group consists of the processes with negligible cross sections and rate coefficients. Processes involving the lowest-lying states (including the ground state) are typically in this group. Adiabatic potentials of the lowest-lying molecular states usually have large energy gaps, so nonadiabatic transition probabilities are very low. The errors in these values are generally harder to evaluate, but in any case these processes are expected to be unimportant for astrophysical applications.

The present findings regarding the cross section and rate coefficient calculations are in agreement with previous conclusions (see, e.g., Belyaev 2013a,b). Naturally, the importance or unimportance of the different processes must ultimately be determined by detailed non-LTE calculations. In any event, the present data provide a reliable basis for accurate non-LTE stellar atmosphere modeling of the spectrum of Si.

Acknowledgements. A.K.B. and S.A.Y. gratefully acknowledge partial support from the Russian Ministry of Education and Science. P.S.B. and A.K.B. acknowledge support from the Royal Swedish Academy of Sciences, the WennerGren Foundation, Göran Gustafssons Stiftelse, and the Swedish Research Council.

\section{References}

Barklem, P. S. 2007, A\&A, 462, 781

Barklem, P. S. 2012, J. Phys.: Conf. Ser., 397, 2049

Barklem, P. S., Belyaev, A. K., \& Asplund, M. 2003, A\&A, 409, L1

Barklem, P. S., Belyaev, A. K., Dickinson, A. S., \& Gadéa, F. X. 2010, A\&A, 519, A20

Barklem, P. S., Belyaev, A. K., Guitou, M., et al. 2011, A\&A, 530, A94

Barklem, P. S., Belyaev, A. K., Spielfiedel, A., Guitou, M., \& Feautrier, N. 2012, A\&A, 541, A80

Belyaev, A. K. 1985, Khimicheskaya Fizika, 4, 750

Belyaev, A. K. 1993, Phys. Rev. A, 48, 4299

Belyaev, A. K. 2013a, Phys. Rev. A, 88, 2704

Belyaev, A. K. 2013b, A\&A, 560, A60

Belyaev, A. K., \& Barklem, P. S. 2003, Phys. Rev. A, 68, 2703

Belyaev, A. K., \& Lebedev, O. V. 2011, Phys. Rev. A, 84, 4701

Belyaev, A. K., \& Tserkovnyi, S. I. 1987, Opt. Spektrosk., 63, 968

Belyaev, A. K., Grosser, J., Hahne, J., \& Menzel, T. 1999, Phys. Rev. A, 60, 2151

Belyaev, A. K., Barklem, P. S., Dickinson, A. S., \& Gadéa, F. X. 2010, Phys. Rev. A, 81, 2706

Belyaev, A. K., Barklem, P., Spielfiedel, A., et al. 2012, Phys. Rev. A, 85, 2704

Bergemann, M., Kudritzki, R.-P., Würl, M., et al. 2013, ApJ, 764, 115

Cohen, J. G., McWilliam, A., Christlieb, N., et al. 2007, ApJ, 659, L161

Croft, H., Dickinson, A. S., \& Gadéa, F. X. 1999a, J. Phys. B: At. Mol. Opt. Phys., 32, 81

Croft, H., Dickinson, A. S., \& Gadéa, F. X. 1999b, MNRAS, 304, 327

Drawin, H. W. 1968, Z. Phys., 211, 404

Drawin, H. W. 1969, Z. Phys., 225, 483

Drawin, H. W., \& Emard, F. 1973, Phys. Lett. A, 43, 333

Fabbian, D., Asplund, M., Barklem, P. S., Carlsson, M., \& Kiselman, D. 2009, A\&A, 500, 1221

Guitou, M., Belyaev, A. K., Barklem, P. S., Spielfiedel, A., \& Feautrier, N. 2011, J. Phys. B: At. Mol. Opt. Phys., 44, 5202

Ji, A. P., Frebel, A., \& Bromm, V. 2014, ApJ, 782, 95

Kalemos, A., Mavridis, A., \& Metropoulos, A. 2002, J. Chem. Phys., 116, 6529

Lambert, D. L. 1993, Phys. Scr., T, 47, 186

Landau, L. D. 1932a, Phys. Z. Sowietunion, 1, 88

Landau, L. D. 1932b, Phys. Z. Sowietunion, 2, 46

Lind, K., Asplund, M., \& Barklem, P. S. 2009, A\&A, 503, 541

Lind, K., Asplund, M., Barklem, P. S., \& Belyaev, A. K. 2011, A\&A, 528, A103

Mashonkina, L. 2013, A\&A, 550, A28

Olson, R. E., Smith, F. T., \& Bauer, E. 1971, Appl. Optics, 10, 1848

Rodionov, D., Belyaev, A. K., Guitou, M., et al. 2014, Izvestia: Herzen University, 165, 63

Shi, J. R., Gehren, T., Mashonkina, L., \& Zhao, G. 2009, A\&A, 503, 533

Shi, J. R., Takada-Hidai, M., Takeda, Y., et al. 2012, ApJ, 755, 36

Steenbock, W., \& Holweger, H. 1984, A\&A, 130, 319

Zener, C. 1932, Proc. Roy. Soc. A, 137, 696 